Copyright (C 2014 IEEE. Personal use of this material is permitted. Permission from IEEE must be obtained for all other uses, in any current or future media, including reprinting/republishing this material for advertising or promotional purposes, creating new collective works, for resale or redistribution to servers or lists, or reuse of any copyrighted component of this work in other works. 


\section{BLIND ESTIMATION OF MIMO RELAY CHANNELS}

\author{
Choo W. R. Chiong, Yue Rong \\ Curtin University \\ Dept. Electrical and Computer Engineering \\ Bentley, WA 6102, Australia
}

\author{
Yong Xiang \\ Deakin University \\ School of Information Technology \\ Melbourne, VIC 3125, Australia
}

\begin{abstract}
In this paper, we integrate two blind source separation (BSS) methods to estimate the individual channel state information (CSI) for the source-relay and relay-destination links of three-node two-hop multiple-input multiple-output (MIMO) relay systems. In particular, we propose a first-order Zdomain precoding technique for the blind estimation of the relay-destination channel matrix, while an algorithm based on the constant modulus and mutual information properties is developed to estimate the source-relay channel matrix. Compared with training-based MIMO relay channel estimation approaches, our algorithm has a better bandwidth efficiency as no bandwidth is wasted for sending the training sequences. Numerical examples are shown to demonstrate the performance of the proposed algorithm.
\end{abstract}

Index Terms - Blind channel estimation, MIMO relay, Z-domain, constant modulus, mutual information.

\section{INTRODUCTION}

For multiple-input multiple-output (MIMO) relay communication systems, the knowledge of the instantaneous channel state information (CSI) is necessary for the retrieval of the source signals at the destination node and the optimization of MIMO relay systems through precoding matrices design and power allocation [1]-[2]. However, the instantaneous CSI is unknown in real wireless communication systems, and thus, has to be estimated at the destination node. One of the possible solutions is by sending known training sequences to assist in the estimation of the instantaneous CSI [3]-[4]. The main problem with the training-based channel estimation algorithms is the high cost involved in sending the training sequences, considering the limited bandwidth available for wireless communication.

In this paper, we propose a blind channel estimation algorithm for three-node two-hop MIMO relay communication systems. This algorithm integrates two different BSS methods

This work was supported under the Australian Research Council's Discovery Projects funding scheme (project numbers DP140102131, DP110102076). to estimate the instantaneous CSI for the individual sourcerelay and relay-destination links. We propose a first-order Zdomain precoding technique for the blind estimation of the relay-destination channel using signals received at the destination node. In this algorithm, the order of the precoders is fixed to one, while a second-order Z-domain precoding algorithm was developed in [5] for blind separation of spatially correlated signals.

With the estimated received signals at the relay node, we propose a blind channel estimation algorithm namely constant modulus and signal mutual information algorithm to estimate the source-relay channel matrix. A similar method was adopted in [6] for the extraction of unknown source signals. We would like to note that algorithms in [5] and [6] were developed for one-hop MIMO systems. Comparing the proposed blind channel estimation algorithm with the trainingbased channel techniques, the former one has a better bandwidth efficiency as all the bandwidth is used for the transmission of the communication signals.

\section{SYSTEM MODEL}

We consider a three-node two-hop MIMO communication system where the source node transmits information to the destination node through a relay node. The source, relay and destination nodes are equipped with $n_{S}, n_{R}$, and $n_{D}$ antennas, respectively. In this paper, we assume that the direct link between the source and destination nodes is sufficiently weak and thus can be ignored. This scenario occurs when the direct link is blocked by obstacles, such as tall buildings or mountains.

The blind channel estimation is completed in two time blocks. In the first time block, the source signals denoted as $\mathbf{s}(n)=\left[s_{1}(n), s_{2}(n), \ldots, s_{n_{S}}(n)\right]^{T}$ are transmitted by the source node. The signals received at the relay node can be expressed as

$$
\mathbf{y}_{r}(n)=\mathbf{H}_{1} \mathbf{s}(n)+\mathbf{v}(n)
$$

where $\mathbf{y}_{r}(n)=\left[y_{r, 1}(n), y_{r, 2}(n), \ldots, y_{r, n_{R}}(n)\right]^{T}$ is the received signal vector, $\mathbf{H}_{1}$ is the $n_{R} \times n_{S}$ channel matrix between the source node and the relay node, $\mathbf{v}(n)=$ 
$\left[v_{1}(n), v_{2}(n), \ldots, v_{n_{R}}(n)\right]^{T}$ is the noise vector at the relay node, and $(\cdot)^{T}$ denotes vector (matrix) transpose.

In the second time block, the received signals are preprocessed by $n_{R}$ first-order precoders $p_{1}(z), p_{2}(z), \ldots, p_{n_{R}}(z)$ as

$$
p_{i}(z)=1-r_{i} z^{-1}, \quad i=1,2, \ldots, n_{R}
$$

where $r_{i}$ is the zero of the precoder $p_{i}(z)$. Note that all zeros are distinct and satisfy $0<\left|r_{i}\right|<1$, for $i=1,2, \ldots, n_{R}$, and are known at the destination node. Here $|\cdot|$ denotes the modulus of a scalar and the determinant of a matrix. The $i$ th coded signal at the relay node can be written as

$$
\begin{aligned}
x_{i}(n) & =\alpha p_{i}(z) y_{r, i}(n) \\
& =\alpha\left[y_{r, i}(n)-r_{i} y_{r, i}(n-1)\right], \quad i=1,2, \ldots, n_{R}
\end{aligned}
$$

where $\alpha>0$ is a scalar adjusting the signal level to satisfy the power constraint at the relay node. The coded signals $\mathbf{x}(n)=\left[x_{1}(n), x_{2}(n), \ldots, x_{n_{R}}(n)\right]^{T}$ are transmitted to the destination node and the received signals at the destination node can be expressed as

$$
\mathbf{y}(n)=\mathbf{H}_{2} \mathbf{x}(n)+\mathbf{w}(n)
$$

where $\mathbf{H}_{2}$ is the $n_{D} \times n_{R}$ channel matrix between the relay node and the destination node and $\mathbf{w}(n)=\left[w_{1}(n), w_{2}(n)\right.$, $\left.\ldots, w_{n_{D}}(n)\right]^{T}$ is the noise vector at the destination node. The transmission power consumed at the relay node can be calculated from (1) and (3) as

$$
\begin{aligned}
& \operatorname{tr}\left(\mathrm{E}\left[\mathbf{x}(n) \mathbf{x}^{H}(n)\right]\right) \\
= & \alpha^{2}\left[\operatorname{tr}\left(\mathbf{P}(z) \mathbf{H}_{1} \mathbf{C}_{\mathbf{s s}}(0) \mathbf{H}_{1}^{H} \mathbf{P}^{H}(z)\right)+\sigma_{v}^{2} \operatorname{tr}\left(\mathbf{P}(z) \mathbf{P}^{H}(z)\right)\right](6)
\end{aligned}
$$

where $\operatorname{tr}(\cdot)$ denotes the matrix trace, $\mathrm{E}[\cdot]$ stands for statistical expectation, $\mathbf{C}_{\mathbf{s s}}(0)=\mathrm{E}\left[\mathbf{s}(n) \mathbf{s}^{H}(n)\right], \mathbf{P}(z)=\operatorname{diag}\left(p_{1}(z)\right.$, $\left.p_{2}(z), \ldots, p_{n_{R}}(z)\right)$ is a diagonal matrix, and $\sigma_{v}^{2}$ is the variance of the noise at the relay node. In this paper, we assume that:

1. All noises are independent and identically distributed (i.i.d.) additive white Gaussian noise (AWGN), and are independent of the source signals.

2. The source signals $s_{1}(n), s_{2}(n), \ldots, s_{n_{S}}(n)$, are temporally white.

3. The number of antennas at the receiving sides are equal or greater than the transmitting sides, i.e., $n_{D} \geq n_{R} \geq n_{S}$.

\section{FIRST-ORDER Z-DOMAIN PRECODING TECHNIQUE}

In this section, we develop a first-order Z-domain precoding algorithm for blind estimation of the relay-destination channel matrix. This blind estimation algorithm aims to find a separation matrix $\mathbf{B}_{1}$ to separate $\mathbf{x}(n)$ and $\mathbf{H}_{2}$ in (5) with only the observable output at the destination node $\mathbf{y}(n)$. Let $\mathbf{B}_{1}=\left[\mathbf{b}_{1,1}, \mathbf{b}_{1,2}, \ldots, \mathbf{b}_{1, n_{R}}\right]$ be an $n_{D} \times n_{R}$ matrix, the desired outcome of the blind channel estimation algorithm is given by

$$
\hat{\mathbf{x}}(n)=\mathbf{B}_{1}^{H} \mathbf{y}(n)=\mathbf{\Lambda} \mathbf{x}(n)+\mathbf{B}_{1}^{H} \mathbf{w}(n)
$$

where $\hat{\mathbf{x}}(n)$ is the estimated coded signals, $(\cdot)^{H}$ denotes complex conjugate transpose, and $\boldsymbol{\Lambda} \triangleq \mathbf{B}_{1}^{H} \mathbf{H}_{2}$ is a diagonal matrix of scaling ambiguity inherited in the blind estimation algorithm. Note that the permutation ambiguity usually associated with BSS does not exist in (7) due to the filtering operation (4) before transmission.

Let us define the autocorrelation matrix of $\mathbf{y}_{r}(n)$ at time lag $k$ as

$\mathbf{C}_{\mathbf{y}_{r} \mathbf{y}_{r}}(k)=\mathrm{E}\left[\mathbf{y}_{r}(n) \mathbf{y}_{r}^{H}(n-k)\right]=\mathbf{H}_{1} \mathbf{C}_{\mathbf{s s}}(k) \mathbf{H}_{1}^{H}+\mathbf{C}_{\mathbf{v v}}(k)$

where $\mathbf{C}_{\mathbf{s s}}(k)$ and $\mathbf{C}_{\mathbf{v v}}(k)$ are the autocorrelation matrices of $\mathbf{s}(n)$ and $\mathbf{v}(n)$, respectively. Note that $\mathbf{C}_{\mathbf{v v}}(k)=\mathbf{0}$ for $k \neq 0$ as the noises are temporally independent. The power spectral matrix of $\mathbf{y}_{r}(n)$ is defined as

$$
\mathbf{Q}_{\mathbf{y}_{r} \mathbf{y}_{r}}(z)=\sum_{k=-\infty}^{\infty} \mathbf{C}_{\mathbf{y}_{r} \mathbf{y}_{r}}(k) z^{-k} .
$$

As the noise covariance matrix at the relay node $\mathbf{C}_{\mathbf{v v}}(0)$ is of full rank, the following proposition is established.

PROPOSITION 1: The power spectral matrix $\mathbf{Q}_{\mathbf{y}_{r} \mathbf{y}_{r}}(z)$ is of full rank at $z=r_{i}$ for $i=1,2, \ldots, n_{R}$.

Let us denote the autocorrelation matrices of $\mathbf{y}(n)$ and $\mathbf{w}(n)$ as $\mathbf{C}_{\mathbf{y y}}(k)$ and $\mathbf{C}_{\mathbf{w w}}(k)$, respectively. It follows from (5) that

$\mathbf{C}_{\mathbf{y y}}(k)=\mathrm{E}\left[\mathbf{y}(n) \mathbf{y}^{H}(n-k)\right]=\mathbf{H}_{2} \mathbf{C}_{\mathbf{x x}}(k) \mathbf{H}_{2}^{H}+\mathbf{C}_{\mathbf{w w}}(k)$

where $\mathbf{C}_{\mathbf{x} \mathbf{x}}(k)$ is the autocorrelation matrix of $\mathbf{x}(n)$ and $\mathbf{C}_{\mathbf{w w}}(k)=\mathbf{0}$ for $k \neq 0$ as the noises are temporally independent. Similarly, the power spectral matrix of $\mathbf{y}(n)$ can be derived based on (3), (9), and (10) as

$$
\begin{aligned}
\mathbf{Q}_{\mathbf{y y}}(z) & =\sum_{k=-\infty}^{\infty} \mathbf{C}_{\mathbf{y y}}(k) z^{-k} \\
& =\mathbf{H}_{2} \mathbf{Q}_{\mathbf{x x}}(z) \mathbf{H}_{2}^{H}+\mathbf{Q}_{\mathbf{w w}}(z) \\
& =\mathbf{H}_{2} \mathbf{P}(z) \mathbf{Q}_{\mathbf{y}_{r} \mathbf{y}_{r}}(z) \mathbf{P}^{H}\left(z^{-1}\right) \mathbf{H}_{2}^{H}+\mathbf{Q}_{\mathbf{w w}}(z)
\end{aligned}
$$

where $\mathbf{Q}_{\mathbf{x x}}(z)$ and $\mathbf{Q}_{\mathbf{w w}}(z)$ are the power spectral matrices of $\mathbf{x}(n)$ and $\mathbf{w}(n)$, respectively.

Let us introduce

$$
\mathbf{T}_{i}(z)=\mathbf{P}_{i}(z) \mathbf{Q}_{\mathbf{y}_{r} \mathbf{y}_{r}}(z) \mathbf{P}^{H}\left(z^{-1}\right), \quad i=1,2, \ldots, n_{R}
$$

where $\mathbf{P}_{i}(z)$ is the matrix $\mathbf{P}(z)$ with the $i$ th diagonal entry replaced by zero, i.e.,

$$
\mathbf{P}_{i}(z)=\operatorname{diag}\left(p_{1}(z), \ldots, p_{i-1}(z), 0, p_{i+1}(z), \ldots, p_{n_{R}}(z)\right) .
$$


It can be deduced that for any $r_{i}$,

$$
\operatorname{rank}\left(\mathbf{P}_{i}\left(r_{i}\right)\right)=n_{R}-1
$$

while the matrix $\mathbf{P}^{H}\left(r_{i}^{-1}\right)$ is of full rank, since $r_{i}^{-1}$ is not a zero of any precoder. It can be further deduced that all elements in the $i$ th row of the matrix $\mathbf{T}_{i}\left(r_{i}\right)$ are zero. Using these results and Proposition 1, the following lemma is established.

LEMMA 1: Matrix $\mathbf{T}_{i}\left(r_{i}\right)$ has a rank of $n_{R}-1$, for $i=$ $1,2, \ldots, n_{R}$, and all rows of matrix $\mathbf{T}_{i}\left(r_{i}\right)$ except for the $i$ th row are linearly independent.

Let $\mathbf{H}_{2, i}$ be equal to $\mathbf{H}_{2}$ with the $i$ th column replaced by a zero vector, i.e.,

$$
\mathbf{H}_{2, i}=\left[\mathbf{h}_{2,1}, \ldots, \mathbf{h}_{2, i-1}, \mathbf{0}, \mathbf{h}_{2, i+1}, \ldots, \mathbf{h}_{2, n_{R}}\right] .
$$

Equation (11) can be rewritten as

$$
\begin{aligned}
\mathbf{Q}_{\mathbf{y y}}\left(r_{i}\right) & =\mathbf{H}_{2} \mathbf{P}\left(r_{i}\right) \mathbf{Q}_{\mathbf{y}_{r} \mathbf{y}_{r}}\left(r_{i}\right) \mathbf{P}^{H}\left(r_{i}^{-1}\right) \mathbf{H}_{2}^{H}+\mathbf{Q}_{\mathbf{w w}}\left(r_{i}\right) \\
& =\mathbf{H}_{2, i} \mathbf{P}_{i}\left(r_{i}\right) \mathbf{Q}_{\mathbf{y}_{r} \mathbf{y}_{r}}\left(r_{i}\right) \mathbf{P}^{H}\left(r_{i}^{-1}\right) \mathbf{H}_{2}^{H}+\mathbf{C}_{\mathbf{w w}}(0) \\
& =\mathbf{H}_{2, i} \mathbf{T}_{i}\left(r_{i}\right) \mathbf{H}_{2}^{H}+\mathbf{C}_{\mathbf{w w}}(0) .
\end{aligned}
$$

Assuming that $\mathbf{C}_{\mathbf{w w}}(0)$ can be estimated and removed from (14), we have

$$
\overline{\mathbf{Q}}_{\mathbf{y y}}\left(r_{i}\right)=\mathbf{H}_{2, i} \mathbf{T}_{i}\left(r_{i}\right) \mathbf{H}_{2}^{H}
$$

The following theorem establishes the estimation criterion for our blind channel estimation algorithm.

THEOREM 1: $\mathbf{b}_{1, i}^{H}$ is a $1 \times n_{D}$ separation vector ensuring

$$
\mathbf{b}_{1, i}^{H} \mathbf{H}_{2}=\left[0, \ldots, 0, c_{i}, 0, \ldots, 0\right]
$$

if and only if

$$
\left\{\begin{array}{l}
\mathbf{b}_{1, i}^{H} \overline{\mathbf{Q}}_{\mathbf{y y}}\left(r_{i}\right)=\mathbf{0} \\
\mathbf{b}_{1, i}^{H} \mathbf{C}_{\mathbf{y y}}(1) \mathbf{b}_{1, i} \neq 0
\end{array}\right.
$$

where $c_{i} \neq 0$ and $i=1,2, \ldots, n_{R}$.

Due to space limit, the proof of Theorem 1 is omitted. We would like to note that theorem 1 holds when the autocorrelation matrix of $\mathbf{y}(n)$ has a time lag of $\tau=1$, i.e., $\mathbf{C}_{\mathbf{y y}}(1)$, and is not valid for other time lag values. Once the separation matrix $\mathbf{B}_{1}$ is obtained, $\mathbf{H}_{2}$ and $\mathbf{y}_{r}(n)$ can be efficiently estimated.

\section{CONSTANT MODULUS AND SIGNAL MUTUAL INFORMATION ALGORITHM}

In this section, the first-hop channel $\mathbf{H}_{1}$ is estimated by applying the proposed constant modulus and signal mutual information algorithm. Define an $n_{R} \times n_{S}$ separation matrix $\mathbf{B}_{2}$ and let

$$
\hat{\mathbf{s}}(n)=\mathbf{B}_{2}^{H} \hat{\mathbf{y}}_{r}(n)=\mathbf{C s}(n)+\mathbf{B}_{2}^{H} \mathbf{v}(n)
$$

where $\hat{\mathbf{y}}_{r}(n)$ is the estimated signals received at the relay node, $\hat{\mathbf{s}}(n)$ is the estimated source signals, and $\mathbf{C} \triangleq \mathbf{B}_{2}^{H} \mathbf{H}_{1}$. This blind channel estimation algorithm aims to obtain the separation matrix $\mathbf{B}_{2}$ in order to recover the first-hop channel $\mathbf{H}_{1}$, only from the estimated relay channel output signals $\hat{\mathbf{y}}_{r}(n)$. Due to the unaccessible source signals, there are two inherent ambiguities in this algorithm, i.e., $\mathbf{C}=\mathbf{B}_{2}^{H} \mathbf{H}_{1}=$ $\mathbf{P} \boldsymbol{\Delta}$, where $\mathbf{P}$ is a permutation matrix and $\boldsymbol{\Delta}$ is a diagonal matrix.

Similar to [6], we propose to exploit the mutual information property of the estimated signals, along with the constant modulus algorithm, to ensure that channel matrices and source signals are completely separated. The following cost function is minimized

$J\left(\mathbf{B}_{2}\right)=\sum_{i=1}^{n_{S}} \mathrm{E}\left[\left(\left|\hat{s}_{i}(n)\right|^{2}-\gamma\right)^{2}\right]+\beta\left[\sum_{i=1}^{n_{S}} \log \left(r_{i i}\right)-\log \left|\mathbf{R}_{\hat{\mathbf{s}} \hat{\mathbf{s}}}\right|\right]$

where $\hat{s}_{i}(n)$ is the $i$ th element of $\hat{\mathbf{s}}(n), \gamma$ is a priori constant dispersion, $\beta$ is a positive real number that balances the constant modulus term and the mutual information term, $r_{i i}$ is the diagonal element of $\mathbf{R}_{\hat{\mathbf{s}} \hat{\mathbf{s}}}$, and $\mathbf{R}_{\hat{\mathbf{s}} \hat{\mathbf{s}}}$ is the covariance matrix of $\hat{\mathbf{s}}(n)$ given by

$$
\mathbf{R}_{\hat{\mathbf{s}} \hat{\mathbf{s}}} \triangleq \mathrm{E}\left[\hat{\mathbf{s}}(n) \hat{\mathbf{s}}^{H}(n)\right] .
$$

From [6], we have the following proposition.

PROPOSITION 2: The mutual information term is zero when $\mathbf{R}_{\hat{\mathbf{s}} \hat{\mathbf{s}}}$ is a diagonal matrix, i.e., when the elements of $\hat{\mathbf{s}}(n)$ are uncorrelated.

Proposition 2 is important to ensure that all source signals are separated from the channel matrix $\mathbf{H}_{1}$ at the destination. The cost function (20) can be rewritten as

$$
\begin{aligned}
& J\left(\mathbf{B}_{2}\right)=\mathrm{E}\left[\sum_{i=1}^{n_{S}}\left(\mathbf{e}_{i}^{T} \mathbf{B}_{2}^{H} \hat{\mathbf{y}}_{r}(n) \hat{\mathbf{y}}_{r}^{H}(n) \mathbf{B}_{2} \mathbf{e}_{i}-\gamma\right)^{2}\right] \\
& +\beta\left[\sum_{i=1}^{n_{S}} \log \left(\mathbf{e}_{i}^{T} \mathbf{B}_{2}^{H} \mathbf{R}_{\hat{\mathbf{y}}_{r} \hat{\mathbf{y}}_{r}} \mathbf{B}_{2} \mathbf{e}_{i}\right)-\log \left|\mathbf{B}_{2}^{H} \mathbf{R}_{\hat{\mathbf{y}}_{r} \hat{\mathbf{y}}_{r}} \mathbf{B}_{2}\right|\right]
\end{aligned}
$$

where $\mathbf{R}_{\hat{\mathbf{y}}_{r} \hat{\mathbf{y}}_{r}} \triangleq \mathrm{E}\left[\hat{\mathbf{y}}_{r}(n) \hat{\mathbf{y}}_{r}^{H}(n)\right]$ is the covariance matrix of $\hat{\mathbf{y}}_{r}(n)$ and $\mathbf{e}_{i}$ is an $n_{S} \times 1$ column vector whose elements are zero except for its $i$ th element which is one.

The gradient of $J\left(\mathbf{B}_{2}\right)$ is given by

$$
\begin{aligned}
\nabla J\left(\mathbf{B}_{2}\right)= & \frac{\partial J\left(\mathbf{B}_{2}\right)}{\partial \mathbf{B}_{2}^{*}} \\
= & 2 \sum_{i=1}^{n_{S}} \mathrm{E}\left[\left(\left|\hat{s}_{i}(n)\right|^{2}-\gamma\right) \hat{\mathbf{y}}_{r}(n) \mathbf{e}_{i}^{T}\left(\hat{\mathbf{y}}_{r}^{H}(n) \mathbf{B}_{2} \mathbf{e}_{i}\right)\right] \\
& +\beta \mathbf{R}_{\hat{\mathbf{y}}_{r} \hat{\mathbf{y}}_{r}} \mathbf{B}_{2}\left[\left(\operatorname{diag}\left(\mathbf{R}_{\hat{\mathbf{s}} \hat{\mathbf{s}}}\right)\right)^{-1}-\mathbf{R}_{\hat{\mathbf{s}} \hat{\mathbf{s}}}^{-1}\right]
\end{aligned}
$$

Then the separation matrix $\mathbf{B}_{2}$ can be obtained by using gradient descent procedure. 


\section{NUMERICAL EXAMPLES}

In this section, we study the performance of the proposed blind MIMO relay channel estimation algorithm through numerical simulations. We consider a three-node two-hop MIMO relay system with $n_{S}, n_{R}$, and $n_{D}$ antennas equipped at the source, relay, and destination node, respectively. The zeros of the precoders are modeled as $r_{i}=\eta_{i} e^{j \pi\left(\frac{2 i-1}{2 n}\right)}$, $i=1,2, \ldots, n_{R}$, where $0<\eta_{i}<1$ and $j=\sqrt{-1}$. This model ensures that the zeros are distinct and satisfy $0<\left|r_{i}\right|<1$, and the angles of zeros are equally spaced on the Z-plane. In the simulations, we assume that all channels are frequency-flat Rayleigh fading, and do not change within one cycle of transmission. All simulation results are averaged over 100 random channel realizations.

We compare the proposed blind estimation algorithm with the training-based MIMO relay channel estimation algorithm developed in [3], where the training sequences are optimized with proper adjustment of simulation parameters for a fair comparison. Fig. 1 shows the normalized mean-squared error (NMSE) performance of estimating $\mathbf{H}_{2}$ and $\mathbf{H}_{1}$ over different values of signal-to-noise ratio (SNR) when $n_{S}=n_{R}=2$, $n_{D}=4$, and the number of samples is chosen as $n=5000$. It can be observed from Fig. 1 that the performance of our proposed algorithm in estimating $\mathbf{H}_{2}$ is comparable to the training-based algorithm even at high SNR. There are error floors in the results of our proposed algorithm due to the estimation of ambiguities existed in the algorithm. At all times, our algorithms have a better bandwidth efficiency compared with channel training algorithm.

The bit-error-rate (BER) performance of two algorithms versus SNR is illustrated in Fig. 2. We also show the BER performance when the channels are perfectly known. It can be seen from Fig. 2 that the performance of our proposed algorithm is very close to the performance of the training-based algorithm. At high SNR, the performances stay constant when the SNR increases as the relay precoding matrix is not optimized in the simulation.

\section{CONCLUSIONS}

We have proposed an integrated blind channel estimation algorithm for three-node two-hop MIMO relay systems. This algorithm can estimate the individual CSI for two-hop MIMO relay systems with a better bandwidth efficiency compared with training-based channel estimation algorithms, as all the bandwidth is used for sending communication signals.

\section{REFERENCES}

[1] X. Tang and Y. Hua, "Optimal design of non-regenerative MIMO wireless relays," IEEE Trans. Wireless Commun., vol. 6, pp. 1398-1407, Apr. 2007

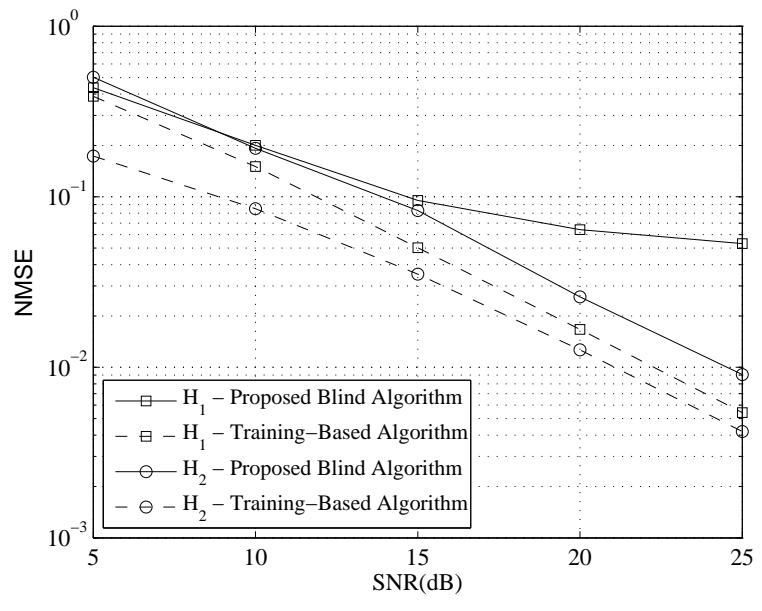

Fig. 1. Normalized MSE versus SNR for $n_{S}=n_{R}=2$, $n_{D}=4$, and $n=5000$.

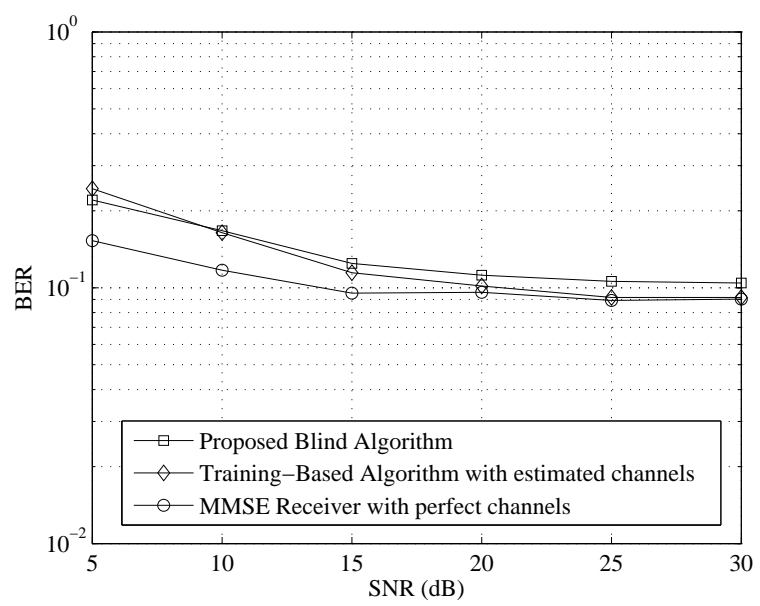

Fig. 2. BER versus SNR for $n_{S}=n_{R}=2, n_{D}=4$, and $n=5000$.

[2] Y. Rong, "Joint source and relay optimization for two-way linear nonregenerative MIMO relay communications," IEEE Trans. Signal Process., vol. 60, pp. 6533-6546, Dec. 2012.

[3] T. Kong and Y. Hua, "Optimal design of source and relay pilots for MIMO relay channel estimation," IEEE Trans. Signal Process., vol. 59, pp. 4438-4446, Sep. 2011.

[4] C. W. R. Chiong, Y. Rong, and Y. Xiang, "Channel training algorithms for two-way MIMO relay systems," IEEE Trans. Signal Process., vol. 61, pp. 3988-3998, Aug. 2013.

[5] Y. Xiang, D. Peng, Y. Xiang, and S. Guo, "Novel Z-domain precoding method for blind separation of spatially correlated signals," IEEE Trans. Neural Networks and Learning Systems, vol. 24, pp. 94-105, Jan. 2013.

[6] Y. Xiang, N. Gu, and K. L. Wong, "Adaptive blind source separation using constant modulus criterion and signal mutual information," in Proc. IEEE Int. Conf. Industrial Technol., pp. 1371-1375, Dec. 2005. 